UCRL-JC-122206

PREPRINT

\title{
Chromosome Translocations Measured by Fluorescence In-Situ Hybridization: A Promising Biomarker
}

\author{
Joe N. Lucas \\ Tore Straume
}

This paper was prepared for the published conference proceedings entitled:

Environmental Toxicology and Risk Assessment: Biomarkers and Risk Assessments: 5th Volume, proceedings from the

American Society for Testing Materials (ASTM) - Fifth Symposium on

Environmental Toxicology and Risk Assessment

Denver, $\mathrm{CO}$

April 2-7, 1995

October 1995

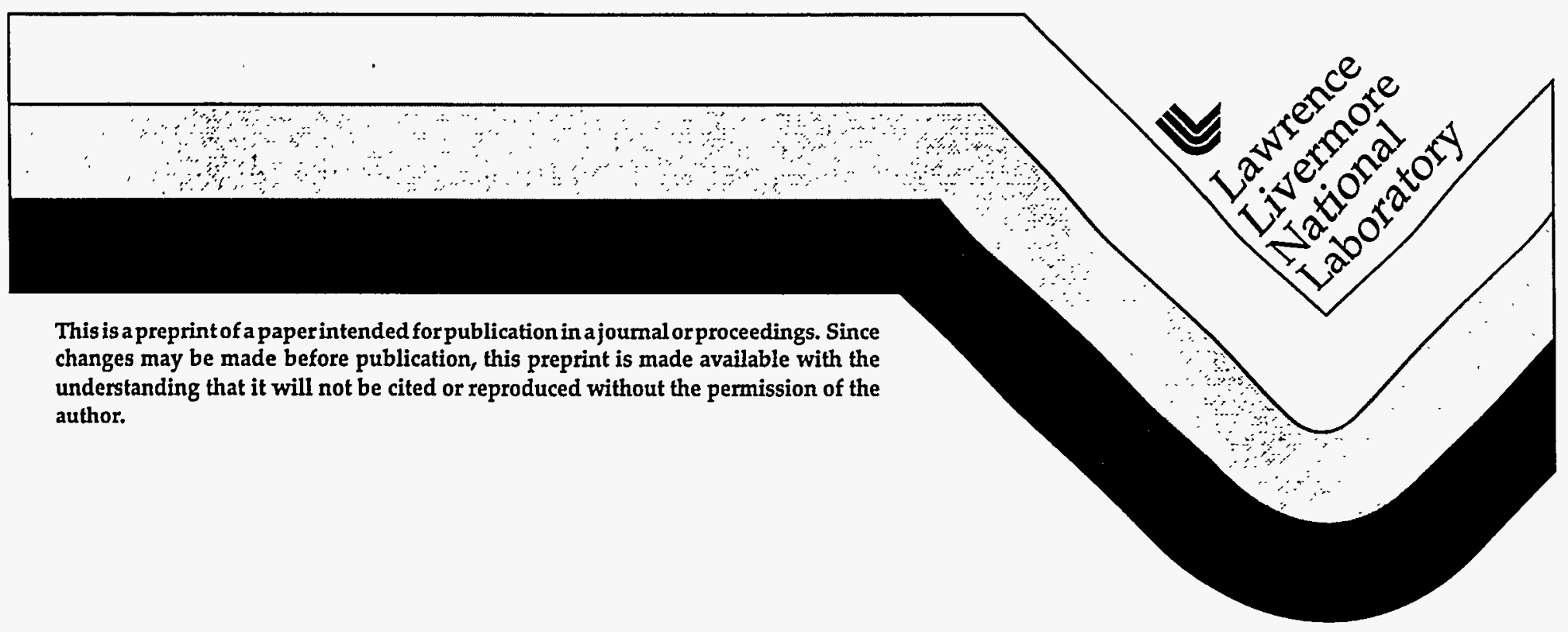




\section{DISCLAIMER}

This document was prepared as an account of work sponsored by an agency of the United States Government. Neither the United States Government nor the University of California nor any of their employees, makes any warranty, express or implied, or assumes any legal liability or responsibility for the accuracy, completeness, or usefulness of any information, apparatus, product, or process disclosed, or represents that its use would not infringe privately owned rights. Reference herein to any specific commercial products, process, or service by trade name, trademark, manufacturer, or otherwise, does not necessarily constitute or imply its endorsement, recommendation, or favoring of the United States Government or the University of California. The views and opinions of authors expressed herein do not necessarily state or reflect those of the United States Government or the University of California, and shall not be used for advertising or product endorsement purposes. 


\section{DISCLAIMER}

Portions of this document may be illegible in electronic image products. Images are produced from the best available original document. 
(Evans et al. 1979). A cocktail of composite chromosome-specific DNA probes are used in combination with pan-centromeric probes to discriminate between reciprocal translocations and dicentrics (e.g., see Lucas et al. 1992a; Straume and Lucas 1993). Chromosome painting is generally performed using DNA probes specific for only a sub-set of the genome, e.g., chromosomes 1, 2, and 4, which in combination results in the detection of $35 \%$ of all translocations. Comparisons with results from conventional cytogenetic methods requires scaling-up the chromosome painting results to full genome equivalents. As described below, we have demonstrated that such scaling can be performed accurately by assuming that radiation results in a random distribution of chromosome breaks.

To visualize interchromosomal exchanges, the metaphase chromosomes are stained yellow with probes for the selected target chromosomes and red for the non-target chromosomes (e.g., see Fig. 1 in Lucas et al. 1992a). With the additional application of blue pan-centromeric probes, the discrimination between translocations and dicentrics is made possible. Thus, exchange aberrations are recognized as bi-color (part red and part yellow) chromosomes, and are scored as reciprocal translocations if the two derivative chromosomes each have one blue-stained centromere and as dicentrics if the derivative chromosomes have two centromeres and a bi-color acentric fragment.

As indicated above, full genomic translocation frequencies are accurately obtained after painting only a small fraction of the genome. This important finding was determined by comparing in the same individuals reciprocal translocation frequencies measured using FISH and G-banding (Lucas et al. 1992a). The frequencies measured using FISH for chromosomes 1,2 and 4 (i.e., $22 \%$ of the genome) were converted to full genome equivalents and then 


\title{
Chromosome Translocations Measured by Fluorescence In-Situ
} Hybridization: A Promising Biomarker

\author{
Joe N. Lucas and Tore Straume \\ Lawrence Livermore National Laboratory, University of California, P.O. Box 808, \\ Livermore, CA 94550.
}

\section{Abstract}

A biomarker for exposure and risk assessment would be most useful if it employs an endpoint that is highly quantitative, is stable with time, and is relevant to human risk. Recent advances in chromosome staining using fluorescence in situ hybridization (FISH) facilitate fast and reliable measurement of reciprocal translocations, a kind of DNA damage linked to both prior exposure and risk. In contrast to other biomarkers available, the frequency of reciprocal translocations in individuals exposed to whole-body radiation is stable with time post exposure, has a rather small inter-individual variability, and can be measured accurately at the low levels. Here, we discuss results from our studies demonstrating that chromosome painting can be used to reconstruct radiation dose for workers exposed within the dose limits, for individuals exposed a long time ago, and even for those who have been diagnosed with leukemia but not yet undergone therapy. 


\section{Introduction}

There is a need for reliable methods to assess past clastogenic exposures and to assess risk. This is particularly the case for a large number of individuals exposed to various levels of ionizing radiation as a result of nuclear accidents such as Chernobyl, atmospheric nuclear testing prior to the early 1960 's, past human experimentation by US Government agencies, the atom bombs dropped on Hiroshima and Nagasaki, various medical radiological procedures, occupational exposures, and a variety of other exposures for which dosimetry information may be poor or absent.

The efforts in our laboratory have centered principally on the development and validation of a technology referred to as "chromosome painting" for use in human exposure and risk assessment. This technology employs fluorescence in situ hybridization (FISH) with whole chromosome probes to rapidly and accurately detect chromosome abnormalities such as stable reciprocal translocations in human cells. The development of this technology began at LLNL during the early 1980's (Pinkel et al. 1986) and has now developed into the method of choice world-wide for the detection of chromosome translocations in humans (e.g., see Lucas et al. 1992a; Nakano et al. 1993; Bauchinger et al. 1993; Straume et al. 1995; National Research Council 1995).

\section{Methodology}

The method employs lymphocytes obtained from a small blood sample taken from the individual to be evaluated. The lymphocytes are cultured and metaphase spreads made on glass slides using standard cytogenetic methods 
plotted against translocation frequencies measured by G-banding for all chromosomes for the same individuals. The results demonstrated that FISH provided reciprocal translocation frequencies that did not differ significantly from those measured by the standard G-banding method.

G-banding, which is universally accepted as an accurate method to detect chromosome translocations, is much too labor intensive for exposure and risk assessment applications. The demonstration that the much faster FISH method provided identical results when scaled to full genome, provided a new practical biomarker for applications that require the scoring of large numbers of cells and individuals.

Much of our recent work has centered on the validation of the FISH technology for radiation dose reconstruction and the development of the data required to translate a measured frequency into a dose. Most of these efforts have been summarized in Straume and Lucas 1995 and are further expanded here.

\section{Human Studies}

To date, we have evaluated four individuals previously exposed to penetrating whole-body radiation, either accidentally or during normal work situations. Each of these four cases were exposed to different kinds of radiation, patterns of exposure, and dose rates. All, however, had in common exceptionally good independent dosimetry against which our biodosimetry results could be compared.

The first case, a DOE radiation worker exposed occupationally during the 1950 's, 1960's, and early 1970s was also evaluated biodosimetrically using 
four different assays, including translocations measured by chromosome painting (Straume et al. 1992). This worker's whole-body, fully penetrating exposure was always within the DOE dose limits of $0.05 \mathrm{~Sv}$ per year. In 1989, the best-estimate dose obtained from the measured translocation frequency was $0.49 \pm 0.21 \mathrm{~Sv}$, in good agreement with the total integrated dose recorded in his official dosimetry record from badge readings of $0.56 \pm 0.20 \mathrm{~Sv}$ (Straume and Lucas 1995). These results suggested that stable biomarkers could be detected in workers, even those exposed within the dose limits.

The second case, a tritium worker in Switzerland, accidentally inhaled tritium oxide in 1985 that resulted in a whole-body dose of $0.44 \mathrm{~Sv}$ based on urinalysis and $0.42 \mathrm{~Sv}$ based on dicentric aberrations measured within one month of the acute inhalation exposure (Lloyd et al. 1986). Our biodosimetry performed for this same individual in 1992 (six years after exposure) using chromosome painting to measure stable reciprocal translocations (Lucas et al. $1992 b)$ resulted in $0.44 \mathrm{~Sv}$, essentially identical to the dosimetry results obtained immediately after the accident from urinalysis and dicentrics (Straume and Lucas 1995).

The third case was also a DOE lab worker exposed to photons and particle radiation from high-energy accelerator operations during 30 -years of work in that environment. This individual was a dosimetry expert and kept meticulous records of his exposure history. His integrated dose-equivalent from personnel dosimeters was $0.33 \pm 0.04 \mathrm{~Sv}$ which compares very well with our biodosimetry results $(0.3 \pm 0.1 \mathrm{~Sv})$ from the translocation frequency in his blood lymphocytes measured during the past year (manuscript in preparation). 
The fourth case was a Ukrainian radiation worker exposed during the past decade to external gamma radiation from $137 \mathrm{Cs}$ and some internal contamination by radiocesium. The external and internal exposure resulted in essentially uniform whole-body radiation dose. Two independent dosimetry methods were employed on this worker: (1) electron paramagnetic resonance (EPR) dosimetry was performed on the individual's tooth enamel by scientists in the Ukraine, and (2) chromosome painting was performed on the individual's blood lymphocytes by us at LLNL. Dosimetry results from the two independent methods show remarkable agreement, i.e., $0.3 \pm 0.1 \mathrm{~Sv}$ for EPR and $0.33 \pm 0.12$ Sv for translocations measured using FISH.

Taken together, these case studies suggest that the frequency of reciprocal translocations in human lymphocytes provide an accurate measure of prior exposure to ionizing radiation in whole-body exposed individuals, regardless of the temporal pattern of the exposure or the kind of radiations involved. Additional individuals with good independent dosimetry are of course being sought to continue these very important validation studies.

Chromosome translocation studies have also been performed on large numbers of individuals such as atom-bomb survivors and criticality accident victims. However, in those cases, the independent dosimetry is uncertain and cytogenetic analyses were not made soon after the radiation exposure. It is nevertheless informative that cytogenetic follow-up for those populations (which unfortunately began many years after the exposure occurred) has shown that the translocation frequencies measured in blood lymphocytes of exposed individuals do not change with time when the same individuals are re-sampled many years later. Individuals and groups from which temporal stability information has been obtained are shown in Fig. 1. It is seen that prior to our 
efforts, there was a multi-year gap in the translocation stability data between exposure and the first measurement.

\begin{tabular}{|lcccc|}
\hline Study Population & $\begin{array}{c}\text { Individuals in } \\
\text { Population }\end{array}$ & \multicolumn{2}{c|}{$\begin{array}{c}\text { Duration of Follow-up } \\
\text { in years }\end{array}$} \\
\hline Hiroshlma/Nagasakl victims & $>100$ & 0 & 10 & 20 \\
\hline Swlss tritlum worker & 1 & 30 & \\
\hline Y-12 Accident victims & 6 & \\
\hline Chernobyl victim & 1 & \\
\hline Rhesus monkeys & 5 & \\
\hline
\end{tabular}

Figure 1. Duration of follow-up in various groups evaluated for chromosome translocations. Plotted from data in Straume and Lucas 1995.

\section{Non-Human Primate Studies}

Rhesus monkeys were exposed in 1965 to whole-body (fully penetrating) radiation in connection with NASA studies (Hardy 1991). Some 28 years later, in 1993, near the end of their lifespan, we performed biodosimetry on blood lymphocytes from six of the primates and compared our results with the actual doses delivered to the animals in 1965 (in one individual, biodosimetry was also performed on skin fibroblasts). Results are listed in Table 1 and show very good agreement between the actual treatment dose given in 1965 and the dose estimated biodosimetrically from the translocations frequency in 1993. It is observed that the biological dose estimates are within $25 \%$ of the actual doses 
for all six animals, and for four of the six animals our biodosimetry differs by less than $10 \%$ from the given doses. Given the fact that almost 30 years had elapsed since exposure, these results are promising indeed.

Table 1. Dose reconstruction for rhesus monkeys irradiated 28 years previously. ${ }^{*}$

\begin{tabular}{cccc}
$\begin{array}{l}\text { Treatment dose } \\
\text { (Sv) }\end{array}$ & $\begin{array}{c}\text { Biodosimetry } \\
\text { dose (Sv) }\end{array}$ & $\begin{array}{c}\text { Biodosimetry dose/ } \\
\text { treatment dose }\end{array}$ & Percent difference \\
\hline 0.56 & 0.59 & 1.05 & 5 \\
1.13 & 0.99 & 0.88 & 12 \\
1.13 & 1.05 & 0.93 & 7 \\
2.25 & 1.70 & 0.76 & 24 \\
2.25 & 2.13 & 0.95 & 5 \\
2.00 & 1.90 & 0.95 & 5 \\
2.25 & 1.84 & 0.82 & 18 \\
\hline
\end{tabular}

"Manuscript in preparation.

\section{In VItro Studies}

The use a biomarker for quantitative evaluation of exposure and dose assessment requires good calibration curves. Such curves provide a relationship between biomarker frequency and dose, and must therefore be obtained for relevant exposure conditions. For radiation-induced chromosome aberrations, calibration curves are generally obtained using human lymphocytes exposed in vitro, which have been shown to be identical to those exposed in vivo (e.g., Brewen and Gengozian 1971). 
Studies currently underway in our program will provide many of the calibration curves necessary for radiation biological dosimetry using the chromosome painting technology. For example, we have recently obtained a full-range calibration curve for 60 Co gamma-ray-induced translocations measured by FISH (Lucas et al. 1995), and we are currently working on a curve for ${ }^{137} \mathrm{Cs}$ gamma rays, which will provide a means for dose reconstruction at Chernobyl where radiocesium contamination is producing most of the population dose. We are also working on calibration curves for tritium beta rays and orthovoltage $x$ rays. This effort is particularly timely now, as the FISH technology is becoming more generally employed in dose reconstruction.

\section{Blodosimetry for Leukemia Patients}

A substantial amount of genetic damage appears in the blood cells of leukemia patients due to the well known phenomenon of genetic instability of cancer cells. This is of particular significance for biological dosimetry because the malignant lymphocytes can mask the genetic damage that may have been caused by radiation or other clastogens prior to the disease. We have developed a new approach that may be used to measure pre-cancer-induced chromosomal aberrations in patients with B-cell leukemia by totally separating the unaffected $T$ lymphocytes from the malignant $B$ lymphocytes (Lucas et al. 1994). Results suggest that pre-leukemia exposure to radiation may be biodosimetrically quantified from translocations frequencies in a certain subset of blood lymphocytes of leukemia patients as long as the blood sample is obtained prior to radio- or chemo-therapy (Lucas et al., 1994). This method fills an important need in the establishment of prior exposures that may have contributed to the induction of leukemia or lymphoma. 


\section{Other Considerations}

Variables such as spontaneous translocation frequencies in unexposed individuals and non-uniform body dose in exposed individuals are also being addressed. We have measured about two dozen unexposed controls of varying ages, from $\sim 20$ to more than 60 years of age. The translocation frequencies range from $\sim 2$ to 10 per 1000 cells (Bender et al. 1988; Straume and Lucas 1995). Further work is needed to fully quantify the effect of variables such as age and natural background radiation.

When the dose distribution is highly non-uniform within the body biodosimetry results can be difficult to evaluate. For example, inhalation or ingestion of radiojodine results in dose primarily to the thyroid. In that case, biodosimetry using blood lymphocytes would not be appropriate. Also, radiation exposures to body extremities such as fingers or hands would result in very few damaged blood lymphocytes. We are working on the development of biodosimetry assays for specific organs that would be particularly useful in assessing partial body exposures.

\section{Conclusion}

When the human case studies are considered together with the results for non-human primates, the overall biodosimetry data are now beginning to provide a strong basis for the use of chromosome translocations detected by FISH in retrospective dosimetry and risk assessment, no matter how long ago the exposure occurred or whether the exposure was received acutely or chronically. 


\section{Acknowledgment}

This work was performed under the auspices of the U. S. Department of Energy by the Lawrence Livermore National Laboratory under contract number W-7405-Eng-48. We are especially grateful for the support of DOE/EH.

\section{References}

Bauchinger, M.; Schmid, E.; Zitzelsberger, H.; Braselmann, H.; Nahrstedt, U. Radiation-induced chromosome aberrations analysed by two-color fluorescence in situ hybridization with composite whole chromosomespecific DNA probes and a pancentromeric DNA probe. Int. J. Radiat. Biol. 64: 179-184 (1993).

Bender, M.A.; Awa, A.A.; Brooks, A.L.; Evans, H.J.; Groer, P.G.; Littlefield, L.G.; Pereira, C.; Preston, R.J.; Wackholtz, B.W. Current status of cytogenetic procedures to detect and quantify previous exposures to radiation. Mutat. Res. 196: 103-159 (1988).

Brewen, J.G.; Gengozian, N. Radiation-induced human chromosome aberrations. II. Human in vitro irradiation compared to in vitro and in vivo irradiation of marmoset leukocytes. Mutat. Res. 13: 383-391 (1971).

Evans, H.C.; Buckton, K.E.; Hamilton, G.E.; Carothers, A., Radiation-induced chromosome aberrations in nuclear-dockyard workers.

Nature 277, 531-534 (1979).

Hardy, K. A. Dosimetry methods used in the studies of the effects of protons on primates: a review. Rad. Res., 126, 120-126 (1991). 
Lucas, J. N., Awa, A., Straume, T., Poggensee, M., Kodama, Y., Nakano, M., Ohtaki, K., Weier, U., Pinkel, D., Gray, J., and Littlefield, G., Rapid translocation frequency analysis in humans decades after exposure to ionizing radiation. Int. J. Radiat. Biol. 62, 53-63 (1992a).

Lucas, J. N., Poggensee, M., Straume, T., The persistence of chromosome translocations in a radiation worker accidentally exposed to tritium. Cytogenet. Cell Genet. 60, 255-256 (1992b).

Lucas, J.N., Straume, T. et al. Discrimination between leukemia- and nonlekemia-induced chromosomal abnormalities in the patient's lymphocytes. Int. J. Radiat. Biol. Int. J. Radiat Biol 66:185-189 (1994).

Lucas, J.N.; Hill, F.; Burk, C.; Fester, T.; Straume, T. Dose-response curve for chromosome translocations measured in human lymphocytes exposed to ${ }^{60} \mathrm{Co}$ gamma rays. Health Phys. 68: $761-765$ (1995).

Nakano, M.; Nakashima, E.; Pawel, D.J.; Kodama, Y.; and Awa, A.; Frequency of reciprocal translocations and dicentrics induced in human blood lymphocytes by $X$-irradiation and determined by fluorscence in situ hybridization. Int. J. Radiat. Biol. 64:565-569 (1993).

National Research Council, Radiation Dose Reconstruction for Epidemiologic Uses. US National Academy of Sciences Press, Washington, DC., 1995, pp. 52-54.

Pinkel, D.; Straume, T.; Gray, J.W. Cytogenetic analysis using quantitative, high sensitivity, fluorescence hybridization. Proc. Natl. Acad. Sci. (USA) 83: 2934-2938 (1986). 
Straume, T., Lucas, J. N., Tucker, J. D., Bigbee, W. L., and Langlois, R. G., Biodosimetry for a radiation worker using multiple assays. Health Phys. 62, $122-130$ (1992).

Straume, T. and J. N. Lucas, A comparison of the yields of translocations and dicentrics measured using fluorescence in situ hybridization. Int. J. Radiat. Biol. 64,185-187 (1993).

Straume, T. and Lucas, J.N. Validation studies for monitoring of workers using molecular cytogenetics. Biomarkers in Occupational Health: Progress and Perspectives (M. L. Mendelsohn, J. P. Peeters, and M. J. Normandt, Eds.), Joseph Henry Press, Washington DC, pp. 174-193 (1995). 